\title{
Les critères de droit utilisés par les juristes pour évaluer l'adéquation de l'information au patient sont-ils médicalement valides?
}

\author{
A propos d'un jugement du tribunal fédéral \\ J.-B. Wasserfallen ${ }^{a}$, A. Telenti ${ }^{b}$, J. Biollaz $^{c}$, A. Crespo ${ }^{d}$
}

\footnotetext{
a Direction médicale

b Division des Maladies Infectieuses

c Division de Pharmacologie Clinique

d Juriste à la Direction du CHUV
}

Correspondance:

Dr Jean-Blaise Wasserfallen

CHUV

Direction médicale

Rue du Bugnon, 46

CH-1011 Lausanne

E-mail: jwasserf@chuv.hospvd.ch

\section{Le contexte}

La question de l'adéquation de l'information donnée au patient est un thème à la mode, à l'origine de plaintes de plus en plus fréquentes auprès des instances judiciaires. Celles-ci utilisent des critères qui leur sont propres pour juger ces affaires. Un jugement du Tribunal Fédéral [1] et une prise de position juridique par rapport à ce jugement [2] font penser que ces critères ne correspondent pas forcément à ceux qui régissent la profession médicale, ce qui est source de malaise et d'incompréhension. Cet article tente de faire le point sur ce cas particulier.

\section{L'histoire clinique}

L'histoire clinique est celle d'une jeune patiente séropositive, traitée par bi-thérapie dès 1996, puis tri-thérapie dès 1997. Ce traitement lui causant des nausées, une inappétence et des vomissements, elle consomme en automédication du Bellergal, à raison de 2 comprimés par jour, médicament prescrit à sa mère et gardé dans la pharmacie familiale. Elle présente alors des paresthésies des membres inférieurs, des myalgies intenses, des vertiges et un malaise diffus qui l'amènent à consulter. La constatation de l'absence de pouls dans ses membres inférieurs motive son hospitalisation en soins intensifs pour ergotisme sévère sur interaction médicamenteuse entre un des médicaments de la trithérapie et le Bellergal. Elle doit finalement être amputée du pied droit et des orteils gauches, et souffre de douleurs séquellaires.

Elle saisit directement le Tribunal Fédéral pour violation du devoir d'information de la part de son médecin traitant au CHUV, employé de l'Etat de Vaud, et réclame un dédommagement qu'elle chiffre à 2,5 millions de francs [3]. Elle obtient gain de cause sur le principe de la responsabilité de l'Etat. Ce montant doit encore faire l'objet d'une décision judiciaire, le Tribunal Fédéral estimant qu'elle est partiellement responsable pour avoir pris un médicament en automédication, et diminuant donc les prétentions de la patiente de $50 \%$.

\section{La procédure judiciaire}

Ce jugement est particulier à plus d'un titre, à commencer par le fait que l'avocate de la patiente a publié un article commentant cette affaire [4]. Il doit avant tout faire réfléchir d'une part le corps médical sur l'étendue de son devoir d'information, et d'autre part les hôpitaux publics sur l'adéquation des procédures de défense qui leurs sont proposées par l'Etat dans ces circonstances.

En premier lieu, la possibilité pour un particulier de saisir directement le Tribunal Fédéral en cas de litige avec un canton n'existe plus. C'est probablement une évolution justifiée, dans la mesure où le système judiciaire, y compris le Tribunal Fédéral, ne saurait prétendre à l'infaillibilité. Or, un jugement au Tribunal Fédéral supprime toute instance de recours. Certaines informations admises lors de ce procès particulier ne sont pas correctes et ont pu exercer une influence non négligeable sur l'issue du jugement, comme on le verra ci-dessous.

\section{Le devoir d'information du médecin et le comportement de son patient: compatibles?}

En ce qui concerne le devoir d'information, il est indéniable que des progrès restent à faire par les médecins, pour permettre au patient d'effectuer un «choix éclairé» [5] et de donner son consentement à des traitements de plus en plus puissants, mais dont les effets indésirables sont également de plus en plus redoutables. Il convient, 
dans ces circonstances, de signaler ces risques, sans alarmer inutilement le patient ou recourir à des procédures exagérément administratives et contraignantes, comme celles proposées dans la prise de position juridique sur ce jugement, récemment publiée [2].

Dans le cas particulier des tri-thérapies, il est important de rappeler que l'introduction du médicament incriminé a suivi une procédure accélérée, sur pression politique. L'ensemble de la procédure a pris 72 jours aux Etats-Unis, un record en la matière [6]. Ces médicaments ont donc été enregistrés sans que les informations habituellement requises pour assurer une certaine sécurité d'emploi ne soient disponibles. Dans la mesure où les effets indésirables n'apparaissent parfois qu'après une large utilisation des médicaments seulement (comme l'a montré l'histoire récente du médicament anti-cholestérol de Bayer), il n'est pas surprenant que des ennuis surviennent, et particulièrement lorsque les procédures visant précisément à garantir la sécurité des médicaments sont court-circuitées. Les médecins (ou l'Etat dans le cadre des hôpitaux publics) ne sauraient être tenus responsables des conséquences de décisions prises au niveau fédéral. Enfin, il est piquant de constater que des patients ont exercé une pression importante pour faire enregistrer ces médicaments, et d'autres maintenant se plaignent d'en subir les effets indésirables.

De plus, la patiente a pris de sa propre initiative un médicament soumis à prescription médicale. Cet accident ne se serait probablement pas produit si le médicament lui avait été prescrit par un médecin et délivré par un pharmacien: ceux-ci auraient tenu compte des autres traitements en cours, et pu théoriquement identifier l'interaction potentielle. Les patients ont du reste la possibilité d'accéder à une information de ce type par le biais des notices contenues dans les emballages des médicaments ou en consultant leur pharmacien, voire Internet, à défaut de leur médecin. La patiente ayant pris ce médicament en dehors du traitement prescrit, sa responsabilité est engagée, comme l'a reconnu le Tribunal Fédéral, en diminuant sa prétention à des dommages intérêts. Nous demeurons toutefois profondément perplexes devant la nécessité reconnue par le Tribunal Fédéral, pour le médecin, d'informer spécifiquement la patiente de cette interaction. Dans quelle mesure un médecin peut-il contrôler tous les actes de ses patients? Et dès lors comment peut-il être tenu responsable des conséquences qui en découlent? Personne n'a jamais, à notre connaissance, attaqué et obtenu des dommages intérêts d'un gara- giste pour lui avoir vendu une voiture de tourisme en cas de dommages survenus dans une utilisation en tout terrain. Toute proportion gardée, il ne saurait y avoir deux poids deux mesures entre ces deux situations.

\section{Les particularités de la responsabilité des médecins des hôpitaux publics}

La responsabilité des médecins travaillant dans les hôpitaux publics est régie par le droit public cantonal, et en droit vaudois, par la Loi vaudoise du 16 mai 1961 sur la responsabilité de l'Etat, des communes et de leurs agents. Son article 4 souligne qu'une faute des agents publics n'est pas exigée: un acte illicite, un dommage et un lien de causalité adéquat suffisent à engager la responsabilité de l'Etat. Dans le cas qui nous occupe, il est indéniable que la patiente a subi un dommage, et qu'un lien de causalité existe entre ce dommage et la consommation de Bellergal en association avec le composant de la tri-thérapie. Restait à démontrer l'illicéité du traitement; il l'est par définition, si l'information fournie n'est pas adéquate. C'est la raison pour laquelle l'ensemble du débat a tourné autour de la question de savoir si le médecin traitant avait spécifiquement mentionné à sa patiente le risque qu'il y avait à prendre sans avis médical un autre médicament en même temps que sa tri-thérapie. Dans le cas précis, mentionner spécifiquement chaque médicament est impossible puisque plus de 150 d'entre eux interagissent avec le même mécanisme de détoxication et sont donc susceptibles de provoquer une interaction médicamenteuse. Dans la mesure où ce médecin ne pouvait fournir la preuve que cette information avait été effectivement donnée, la parole de la plaignante l'emportait, en vertu du principe du renversement du fardeau de la preuve, qui prévaut actuellement dans ces cas de litige.

A ce stade, il convient de signaler que l'information sur l'interaction médicamenteuse en question n'était pas disponible à l'époque, comme l'atteste l'expertise extra-judiciaire demandée dans ce cas. Cette malheureuse histoire a d'ailleurs contribué, en même temps qu'un ou 2 autres cas rapportés dans la littérature, à la modification du texte d'accompagnement du médicament antiviral incriminé (à noter que la notice du Bellergal n'a été modifiée elle qu'en 1999, soit 2 ans plus tard!). De plus, la carte d'information distribuée par le fabricant du médicament anti-viral n'existait qu'en français, et n'était disponible que depuis l'été 1997 (soit après l'accident), et non pas depuis 1996, comme 
mentionné dans les considérants du jugement [1]. L'affaire a donc été jugée sur la base d'informations inexactes. La question se pose alors de savoir dans quelle mesure l'Etat a été adéquatement défendu dans cette affaire.

La reprise des considérants du jugement montre que l'audience d'administration des preuves a réuni l'expert de la $\mathrm{FMH}$, le médecin traitant et le chef de clinique de l'hôpital. A aucun moment, des experts des maladies infectieuses ou de pharmacologie clinique de l'hôpital n'ont été sollicités pour exprimer leur point de vue. Dans une telle circonstance, l'Etat devrait parfaitement pouvoir faire appel aux spécialistes de son choix pour maximiser ses chances d'éviter une condamnation. C'est la raison pour laquelle, suite à ce jugement, la Direction de l'hôpital a décidé de modifier ses procédures et de faire examiner systématiquement par la Direction médicale toutes les plaintes en responsabilité civile ou pénale reçues par son juriste. Il sera ensuite décidé de cas en cas de solliciter l'avis des spécialistes du domaine concerné, et de les faire citer comme témoins à l'audience.

\section{L'impact de ce jugement sur l'activité médicale}

Sur un plan local, en plus de la modification de stratégie décrite ci-dessus, ce jugement a conforté la Direction de l'hôpital à poursuivre son effort dans le domaine de l'information au patient, pour inciter ses services à rédiger et utiliser des feuilles d'information écrites, sur lesquelles est recueillie ou non la signature du patient, mais qui sont systématiquement distribuées. Cela devrait ainsi constituer la preuve que l'information a été donnée, à la satisfaction des juristes.

Parallèlement, l'établissement a décidé de modifier ses ordonnances et d'y inclure la mention que la consommation de tout médicament en dehors du traitement prescrit peut comporter des risques d'atteinte à la santé.

Toute cette affaire, qui va avoir un impact important sur la relation médecin-patient, n'estelle pas regrettable? En effet, après avoir été longtemps paternaliste, cette relation évoluait vers un partenariat. Ce jugement montre que cette perspective n'est pas réaliste. La seule réponse possible ne sera-t-elle pas de recourir de manière accrue à des documents écrits, pour lesquels la signature du patient sera requise? C'est d'ailleurs ce que préconise un juriste dans un article récent sur la même affaire, paru en allemand dans le Bulletin des Médecins Suisses [2]: 1) le devoir d'information ne se limite pas aux procédures invasives; 2) les dangers de l'automédication doivent être inclus dans l'information; 3) celle-ci doit être documentée, en impliquant des témoins; 4) la sécurité absolue n'existant pas, plus l'information donnée est protocolée de manière exhaustive, plus elle a de chance de satisfaire aux critères des juristes. Cette activité, qui prend énormément de temps, n'est actuellement pas rémunérée. C'est bien une preuve que les critères du droit ne sont pas médicalement valides. N'entre-t-on pas ainsi de plein pied dans une médecine bureaucratique, procédurière et défensive, qui non seulement ne va pas contribuer à en diminuer les coûts, mais, plus dommageable encore, fait fi de la clé de voûte de la relation médecin-malade, à savoir la confiance?

\section{Références}

1 Jugement du 27 novembre 2001. Http://www.bger.ch (mot-clé: jurisprudence, arrêts dès 2000: interaction).

2 Conti C. Aufklärungspflicht bei Medikamentenabgabe: neues Bundesgerichtsurteil. Bull Méd Suisses 2002;83:1185-6.

3 Bolliger L. Tribunal Fédéral: les juges lui donnent raison. Elle réclame 2,5 millions au CHUV. 24 Heures, 28.11.2001.

4 Cereghetti A. Quelles sont l'étendue et la nature du devoir d'information des médecins? Le Temps, 8.3.2002.

5 Kuhn HP. Information pré-opératoire: un devoir d'optimisation. Bull Méd Suisses 2000;81:2180-93.

6 Cimons M. FDA moves quickly, oks new drug to fight AIDS medicine: Agency also licenses ritonavir for use in early stages of infection. Action exceeds recommendation. Los Angeles Times, 2.3.1996. 